\title{
Greater Pain Severity is Associated with Inability to Access Addiction Treatment Among a Cohort of People Who Use Drugs
}

This article was published in the following Dove Press journal: Journal of Pain Research

\author{
Pauline Voon ${ }^{1,2}$ \\ Linwei Wang ${ }^{3,4}$ \\ Ekaterina Nosova (D) ${ }^{\prime}$ \\ Kanna Hayashi iD ${ }^{1,5}$ \\ Michael John Milloy ${ }^{1,6}$ \\ Evan Wood ${ }^{1,6}$ \\ Thomas Kerr ${ }^{1,6}$
}

'British Columbia Centre on Substance Use, Vancouver, BC V6Z 2A9, Canada;

${ }^{2}$ School of Population and Public Health, University of British Columbia,

Vancouver, BC V6T IZ3, Canada; ${ }^{3}$ British

Columbia Centre for Excellence in HIV/ AIDS, St Paul's Hospital, Vancouver, BC V6Z IY6, Canada; ${ }^{4}$ Centre for Urban Health Solutions, St Michael's Hospital, Li Ka Shing Knowledge Institute, Toronto, ON M5B IT8, Canada; ${ }^{5}$ Faculty of Health Sciences, Simon Fraser University, Burnaby, BC V5A IS6, Canada;

${ }^{6}$ Department of Medicine, University of British Columbia, Vancouver, BC V6Z 2A9, Canada

\begin{abstract}
Aim: Given that co-occurring pain is prevalent among people who use drugs (PWUD), we sought to explore the effect of pain severity on accessing addiction treatment.

Methods: Data were derived from two prospective cohort studies of PWUD in Vancouver, Canada from June 2014 to May 2016. Multivariable generalized linear mixed-effects multiple regression (GLMM) analyses were used to investigate the association between average pain severity and self-reported inability to access addiction treatment.
\end{abstract}

Results: Among 1348 PWUD, 136 (10.1\%) reported being unable to access addiction treatment at least once over the study period. Individuals who reported being unable to access addiction treatment had a significantly higher median average pain severity score (median=5, IQR=0-7) compared to individuals reporting no inability to access addiction treatment (median $=3, \mathrm{IQR}=0-6, p=0.038$ ). Greater pain severity was independently associated with higher odds of reporting inability to access addiction treatment (AOR: 1.75, 95\% CI: $1.08-2.82$ for mild-moderate vs no pain; AOR: $1.98,95 \%$ CI: $1.27-3.09$ for moderatesevere vs no pain).

Conclusion: PWUD with greater pain severity may be at higher risk of being unable to access addiction treatment, or vice versa. While further research is needed to confirm causal associations, these data suggest that there may be underlying pathways or mechanisms through which pain may be associated with access to addiction treatment for PWUD.

Keywords: pain, addiction, substance use, health services, opioid agonist treatment, methadone

\section{Plain Language Summary}

Pain is common among people who use drugs, yet the effect of pain on the ability for this population to access addiction treatment has not yet been thoroughly explored. We examined data from two prospective cohort studies of people who used drugs in Vancouver, Canada from June 2014 to May 2016. Among 1348 people who used drugs, $10 \%$ reported inability to access addiction treatment, and $75 \%$ reported pain at least once during the study period. We found that people with more severe pain were significantly more likely to report that they were unable to access addiction treatment. Participants with the highest pain severity were nearly twice as likely to report inability to access addiction treatment compared to participants reporting no pain. These results suggest that, among people who use drugs, pain severity and access to addiction treatment may be related to each other, although further research is needed to fully understand how these factors may influence one other.
Correspondence: Thomas Kerr Department of Medicine, University of British Columbia, 400-1045 Howe Street, Vancouver, BC V6Z 2A9, Canada

$\mathrm{Tel}+\mathrm{I} 778945-7616$

$\mathrm{Fax}+1604$ 428-5183

Email bccsu-tk@bccsu.ubc.ca 


\section{Introduction}

The crisis of opioid-related overdose continues to devastate communities across North America. ${ }^{1}$ Given that opioid addiction has been recognized as a major risk factor for morbidity and mortality, there is an urgent need to expand access to addiction treatment. ${ }^{2}$ People who use drugs (PWUD) demonstrate a high prevalence of cooccurring pain, ${ }^{3}$ and pain in this population may be associated with poorer addiction treatment outcomes such as increased risk of relapse ${ }^{4}$ and lower rates of retention in treatment and abstinence. ${ }^{5}$ Despite this, the impact of pain on the ability of PWUD to access addiction treatment has not been thoroughly investigated. Therefore, this study seeks to investigate the association between pain severity and ability to access addiction treatment among PWUD.

\section{Methods}

This study utilizes data from two ongoing prospective cohort studies of PWUD in Vancouver, Canada: the Vancouver Injection Drug Users Study (VIDUS) and the AIDS Care Cohort to evaluate Exposure to Survival Services (ACCESS). ${ }^{6}$ These cohorts recruit participants through community-based methods such as street outreach. Individuals are eligible for the studies if they are at least 18 years of age; have injected illicit drugs in the month prior to enrollment (VIDUS) or used an illicit drug other than, or in addition to, cannabis in the month prior to enrollment (ACCESS); and are HIV-seronegative (VIDUS) or HIV-seropositive (ACCESS). Participants provide written informed consent, answer questionnaires administered by an interviewer at baseline and semi-annual visits, and receive a \$40 (CAD) stipend at the end of each visit. While participants are encouraged to complete follow-up interviews every six months, the timing of interviews may vary depending on when individuals return for their follow-up interviews, which may not take place at exactly six months after their previous interview. In other words, participants are eligible to return for a follow-up interview so long as at least six months have passed since their last interview. These studies receive ethical approval from the University of British Columbia and Providence Health Care Research Ethics Boards.

The present study includes data from the intervieweradministered questionnaires that were conducted between June 1, 2014 (the date at which the Brief Pain Inventory was first included in the cohort questionnaires) and May 31, 2016. Observations with missing responses to the primary outcome and explanatory variables of interest were excluded. The primary dependent variable was inability to access addiction treatment, which was ascertained using the question: "In the last six months, have you ever tried to access any drug/alcohol treatment program but were unable to?" (yes vs no).

The primary independent variable was average pain severity, which was derived using the zero-to-10 average past-week pain scale of the Brief Pain Inventory Short Form (BPI-SF). To assess dose-response effects, average pain severity was initially categorized into none $(0)$, mild (1-4), moderate (5-6), and severe (7-10), which have been identified as optimal cut-points in the literature. ${ }^{7}$ However, due to small cell counts for the outcome in the mild pain category when using these cut-points (inability to access addiction treatment: $n=46,24,46$, and 48 for no, mild, moderate, and severe pain, respectively), the moderate pain category was collapsed in order to spread its sample size evenly across categories, and the cut-points redefined as: none (0), mild-moderate (1-5), and moderatesevere $(6-10)(n=46,48$, and 70 , respectively, reporting inability to access addiction treatment). To assess linear trends with pain severity, the models were replicated with pain severity as a numeric (ie, noncategorical) variable.

Potentially confounding variables, which were identified based on conceptual and demonstrated associations with pain and inability to access addiction treatment, ${ }^{8}$ included: age (per year older); HIV serostatus (positive vs negative); hepatitis $\mathrm{C}$ serostatus (positive vs negative); gender (male vs female or other); ethnicity (white vs other); homelessness (yes vs no); highest education completed ( $\geq$ vs $<$ high school completion); incarceration (yes vs no); having been attacked, having been assaulted, or having suffered from violence (yes vs no); having ever been diagnosed with a mental illness (yes vs no); nonfatal overdose (yes vs no); heroin use ( $\geq$ vs $<$ daily); prescription opioid use $(\geq$ vs $<$ daily); cocaine or crack use ( $\geq$ vs $<$ daily; collapsed into one variable due to small frequencies when disaggregated); methamphetamine use ( $\geq$ vs $<$ daily); and heavy alcohol use (more than four drinks per day or 14 drinks per week for men, or more than three drinks per day or seven drinks per week for women). The covariate of having been attacked, having been assaulted, or having suffered from violence was selected as a potential confounder given its demonstrated association with pain and inability to access addiction treatment in other research, ${ }^{8}$ as well as on the basis of some clinical policies in which a patient demonstrating violence may be "red flagged," discharged, banned, or otherwise managed in ways that may affect the patient's 
access to health services. ${ }^{9-11}$ The recall period for these variables was the six months before the interview visit, unless indicated otherwise. Current enrollment in addiction treatment was not included as a confounder as it did not show statistical significance. Furthermore, because the recall period for inability to access addiction treatment was defined as any point within the six months prior to a participant's interview, the sample was not restricted to individuals who were not enrolled in addiction treatment, given the possibility that an individual could have initially been unable to access treatment at some point prior to successful enrollment.

Descriptive characteristics of the study sample were examined using Pearson's chi-squared tests and Mann-Whitney $U$-tests. Then, we used generalized linear mixed-effects models (GLMMs) with logit link and binomial distribution to determine the effect of pain on inability to access addiction treatment. ${ }^{12}$ Random intercepts were applied to account for random variation and repeated measurements from the same participants over time. The random intercept that was specified in the model functioned to account for unmeasured individualspecific confounding that is fixed over time from individuals who contributed to multiple observations during the study period. ${ }^{13}$ Bivariable GLMMs were examined between the dependent and independent variables, from which significant $(p \leq 0.1)$ variables were used in a multivariable confounding GLMM ${ }^{14}$ Unless indicated otherwise, two-sided $p$-values were used, and $p<0.05$ was defined as statistical significance. $\mathrm{R}$ version 3.1.1 (R Foundation for Statistical Computing, Vienna, Austria, 2016) was used for all analyses.

\section{Results}

During the study period, 1348 participants completed at least one study interview and were eligible for inclusion in this analysis. After excluding 38 observations with missing responses to the primary dependent or independent variables, participants in this sample contributed 4240 complete observations with a median of four (IQR: 3-4) follow-up visits. At the start of the study period, the total study sample was $64.6 \%$ male, $55.4 \%$ white, and had a median age of 49 years (IQR: $41-55$ years). The median average pain severity score at baseline for the total sample was 3 (IQR: 0-6). In total, $136(10.1 \%)$ participants reported inability to access addiction treatment at least once during the study period, and 1008 (74.8\%) participants reported an average pain severity of $\geq 1$ at least once during the study period.

Figure 1 shows the distribution of average pain severity scores stratified by inability to access addiction treatment.

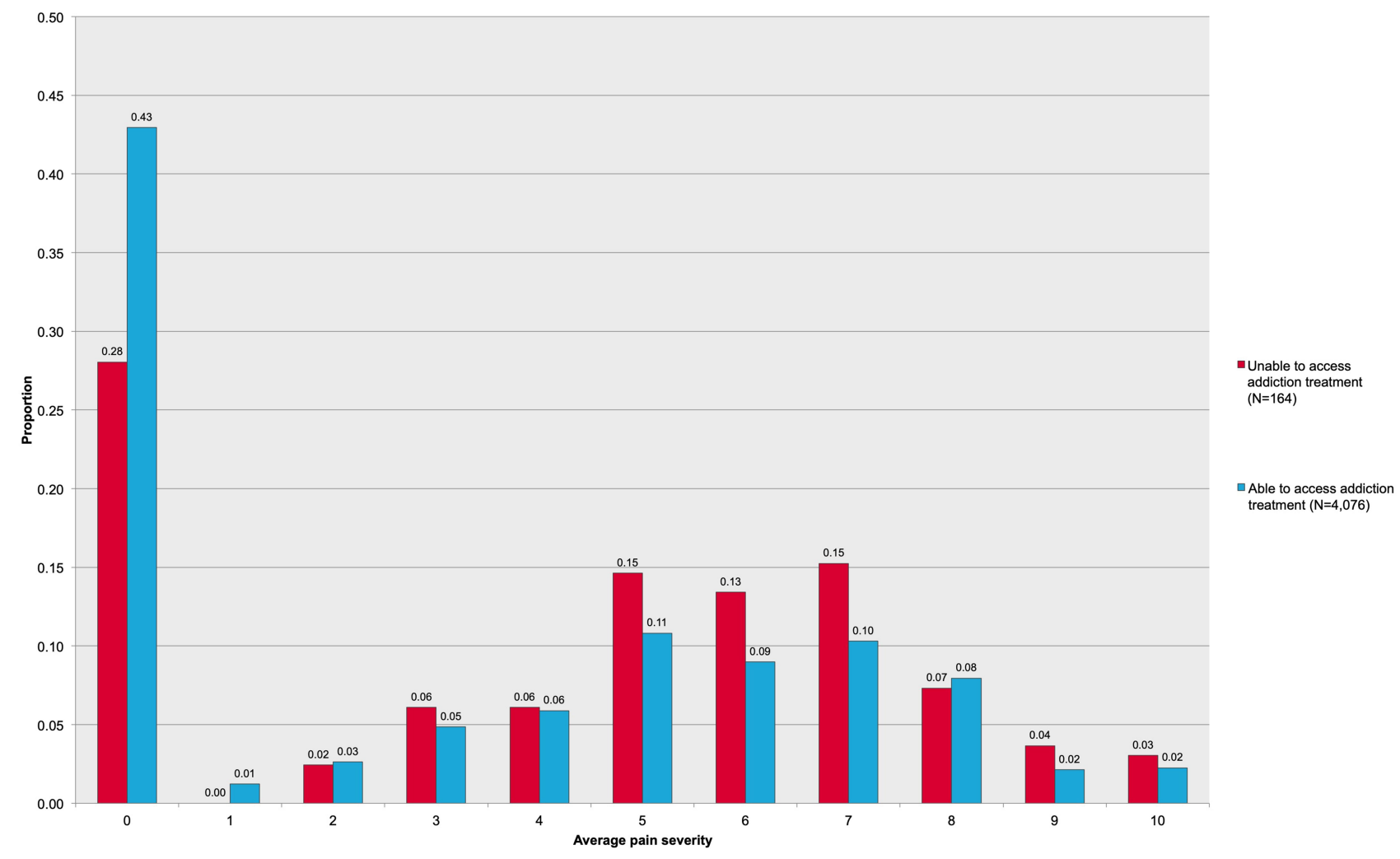

Figure I Distribution of average pain severity scores stratified by ability to access addiction treatment ( $\mathrm{n}=1348$ contributing to 4240 observations). 
Table I Bivariable and Multivariable Generalized Linear Mixed Multiple Regression Analyses of Effect of Average Pain Severity on Selfreported Inability to Access Addiction Treatment ${ }^{a}$ Among People Who Use Drugs $(n=1348)$

\begin{tabular}{|l|l|l|l|l|}
\hline \multicolumn{2}{|l|}{ Unadjusted } & \multicolumn{2}{l|}{ Adjusted $^{\mathbf{a}}$} \\
\hline Average Pain Severity & Odds Ratio (95\%Cl) & p-value & \multicolumn{2}{l|}{ Odds Ratio (95\%Cl) $^{\mathbf{b}}$} \\
\hline None (reference) & & & & \\
Mild-moderate (I-5) & $1.74(1.07-2.83)$ & 0.027 & $1.75(1.08-2.82)$ & 0.023 \\
Moderate-severe (6-10) & $2.17(1.37-3.43)$ & 0.001 & $1.98(1.27-3.09)$ & 0.003 \\
\hline
\end{tabular}

Notes: ${ }^{a}$ Adjusted for confounders found to be significant at the level of $p \leq 0.1$ in the bivariable analyses (ie, age, ethnicity, homelessness, incarceration, violence, mental illness, overdose, heroin use, prescription opioid use, methamphetamine use, and heavy alcohol use). Activities/events refer to the six months prior to the participant's interview. ${ }^{b}$ Average pain severity estimates as a numeric (ie, noncategorical) variable: unadjusted $\mathrm{OR}=\mathrm{I} .1 \mathrm{I}, 95 \% \mathrm{Cl}$ : I.04-I.I8, $p<0.00 \mathrm{I}$; AOR (adjusted for same covariates as above adjusted model) $=1.10,95 \% \mathrm{Cl}$ : I.04-I. $17, p=0.002$.

Participants reporting inability to access addiction treatment displayed a significantly higher median average pain severity (median $=5, \mathrm{IQR}=0-7$ ) compared to participants reporting no inability to access addiction treatment (median=3, IQR $=0-6, p=0.038$ ).

As indicated in Table 1, a dose-response relationship was observed between higher average pain severity and inability to access addiction treatment in both bivariable and multivariable analyses. After controlling for significant confounders, the adjusted odds of reporting inability to access addiction treatment increased from 1.75 (95\%CI: $1.08-2.82)$ to 1.98 (95\% CI: 1.27-3.09) for mild-moderate and moderate-severe average pain, respectively. Likewise, using numeric pain severity in the trend test, a significant association was observed with inability to access addiction treatment in both bivariable (OR: 1.11, 95\%CI: 1.04-1.18) and multivariable analyses (AOR: $1.10,95 \%$ CI: $1.04-1.17)$ adjusted for the same covariates as the categorical pain model.

\section{Discussion}

In this study of 1348 people who use illicit drugs, 10.1\% reported inability to access addiction treatment, and $74.8 \%$ reported pain at least once during the study period. Average pain severity was associated with increased odds of reporting inability to access addiction treatment in a dose-response relationship. In the highest pain severity category, participants reported inability to access addiction treatment at nearly twice the odds compared to participants reporting no pain. Median average pain severity scores were also significantly higher for participants reporting inability to access addiction treatment. These results suggest that, among PWUD, higher pain severity appears to be significantly associated with inability to access addiction treatment.

The dose-response relationship between greater pain severity and inability to access addiction treatment in this study is concerning. Notably, the outcome being examined was the inability to access addiction treatment despite having tried to access such services, suggesting that individuals were actively seeking treatment but were faced with barriers when they attempted to do so. In light of the ongoing opioid overdose crisis, reducing barriers and increasing accessibility to addiction treatment is an urgent public health priority. In this regard, these findings suggest that pain may pose a significant barrier to accessing addiction treatment for PWUD. While there is a paucity of other literature on the role of pain in accessing addiction treatment, other studies have found that pain is a significant concern among individuals seeking addiction treatment, ${ }^{5,15-18}$ and that persistent pain among individuals enrolled in addiction treatment is associated with increased likelihood of substance use severity and frequency, positive urine drug screens, shorter treatment duration, hospitalization, and relapse; decreased likelihood of abstinence; and higher costs associated with health service utilization (ie, inpatient, medical, psychiatric, and other service costs). ${ }^{5,19-21}$ Pain may present a significant challenge to accessing addiction treatment due to the scarcity of evidence-based guidelines for pain management among individuals in addiction treatmentparticularly for individuals on opioid agonist treatment who have concurrent pain and may require significantly higher doses compared to individuals without concurrent pain. ${ }^{22-25}$ Other possible reasons why pain may be associated with inability to access addiction treatment may include clinic policies that may prohibit the treatment of non-addiction related health concerns; physical mobility or geographic barriers that may limit the ability of individuals experiencing pain to access treatment services; stigma or past negative healthcare experiences related to pain or substance use; or long wait times that may be difficult for a person experiencing pain to endure. ${ }^{8,26,27}$ Further research is needed to explore the underlying pathways or mechanisms through which pain may be associated with accessibility of addiction treatment. 
There are limitations to this study that should be noted. The use of self-reported data in this study is subject to socially desirable reporting and recall bias. In addition, the outcome of interest categorized people who attempted to access addiction treatment but were unable to, and compared these individuals both to those who did not attempt to access treatment, as well as those who accessed treatment without barriers. Thus, the effect of pain on addiction treatment need or uptake could not be separately examined in this study. Furthermore, the present analysis was intentionally not restricted to individuals with chronic or persistent pain only, as access to addiction treatment may be influenced both by acute or chronic pain equally. However, acute vs chronic pain may be addressed differently in different treatment settings, which may result in different barriers to accessing treatment depending on the type of pain manifested, although such differences could not be discerned in this study. The temporal relationships underlying the association between pain and inability to access addiction treatment also cannot be fully understood from this analysis, particularly as it was not explicitly asked in the questionnaire whether pain was a reason for inability to access addiction treatment, and we compared past-week pain severity to pastsix month inability to access addiction treatment. While studies have found that past-week pain severity scores are similar to pain scores obtained using longer reference periods, ${ }^{28}$ there is potential for bias in this study due to the differing recall periods of the variables under investigation. Finally, these results may not be generalizable to other populations of PWUD in other settings in which accessibility of health services and addiction treatment may differ, especially since the present study's setting included universal, no-cost access to medical care and primary care clinics that endorse harm reduction.

\section{Conclusion}

In this study of PWUD, higher pain severity was significantly and independently associated in a dose-response relationship to higher odds of reporting inability to access addiction treatment. Based on these results, we infer that among PWUD, pain may be an important factor warranting assessment and treatment given its potential association with inability to access addiction treatment, particularly among a population at high risk for morbidity and mortality related to self-medication of pain. At the same time, efforts to improve access to addiction treatment may assist PWUD in having pain management and other medical needs addressed, thereby lessening the potential for pain-related risks and harms among this population.

\section{Abbreviations}

ACCESS, AIDS Care Cohort to evaluate Exposure to Survival Services; BPI-SF, Brief Pain Inventory Short Form; CDN, Canadian dollar; AOR, adjusted odds ratio; HIV, human immunodeficiency virus; GLMM, generalized linear mixed-effects model; PWUD, people who use drugs; VIDUS, Vancouver Injection Drug Users Study.

\section{Ethics Approval and Informed Consent}

The ACCESS and VIDUS studies receive ethical approval from the University of British Columbia and Providence Health Care Research Ethics Boards. All participants provide written informed consent prior to their participation in the studies.

\section{Acknowledgments}

The authors wish to thank the study participants for their contribution to the research, as well as current and past researchers and staff.

\section{Author Contributions}

All authors made substantial contributions to conception and design, acquisition of data, or analysis and interpretation of data; took part in drafting the article or revising it critically for important intellectual content; agreed to submit to the current journal; gave final approval of the version to be published; and agree to be accountable for all aspects of the work.

\section{Funding}

This study was supported by the US National Institutes of Health (U01DA038886 and U01DA021525) and the Canadian Institutes of Health Research through the Canadian Research Initiative on Substance Misuse (SMN-139148). This research was undertaken, in part, thanks to funding from the Canada Research Chairs program through a Tier 1 Canada Research Chair in Inner City Medicine which supports Dr Evan Wood. Pauline Voon is supported through a Vanier Canada Graduate Scholarship from the Canadian Institutes of Health Research (CIHR) and a Doctoral Scholarship from The Pierre Elliott Trudeau Foundation. Dr Kanna Hayashi is supported by a CIHR New Investigator Award (MSH141971), a Michael Smith Foundation for Health Research (MSFHR) Scholar Award, and the St Paul's Foundation. M-J Milloy is supported by the United States National Institutes of Health (U01-DA0251525), 
a New Investigator award from the Canadian Institutes of Health Research, and a Scholar Award from the Michael Smith Foundation for Health Research.

\section{Disclosure}

Pauline Voon reports funding from the Canadian Institutes of Health Research and the Pierre Elliott Trudeau Foundation during the conduct of the study. M-J Milloy is the Canopy Growth Professor of Cannabis Science at the University of British Columbia (UBC), a position created using unstructured arms' length gifts to the university from Canopy Growth Corporation, a licensed producer of cannabis, and the Government of British Columbia's Ministry of Mental Health and Addictions; and is supported by the United States National Institutes of Health (U01-DA0251525), a New Investigator Award from CIHR and a Scholar Award from the Michael Smith Foundation for Health Research (MSFHR). UBC has also received unstructured funding from NG Biomed, Ltd, an applicant to the Canadian Federal Government for a license to produce cannabis, to support M-JM. The funders had no role in study design, data collection and analysis, decision to publish, or preparation of the manuscript. M-JM has no personal financial relationships with the cannabis industry. Thomas Kerr reports grants from the US National Institutes of Health and the Canadian Institutes of Health Research during the conduct of the study. The authors report no other potential conflicts of interest in this work.

\section{References}

1. Volkow ND, Frieden TR, Hyde PS, Cha SS. Medication-assisted therapies-tackling the opioid-overdose epidemic. $N$ Engl $J$ Med. 2014;370(22):2063-2066. doi:10.1056/NEJMp1402780

2. Kolodny A, Courtwright DT, Hwang CS, et al. The prescription opioid and heroin crisis: a public health approach to an epidemic of addiction. Annu Rev Public Health. 2015;36:559-574. doi:10.1146/annurevpublhealth-031914-122957

3. Voon P, Karamouzian M, Kerr T. Chronic pain and opioid misuse: a review of reviews. Subst Abuse Treat Prev Policy. 2017;12(1):36. doi:10.1186/s13011-017-0120-7

4. Worley MJ, Heinzerling KG, Shoptaw S, Ling W. Pain volatility and prescription opioid addiction treatment outcomes in patients with chronic pain. Exp Clin Psychopharmacol. 2015;23(6):428-435. doi: $10.1037 /$ pha0000039

5. Caldeiro RM, Malte CA, Calsyn DA, et al. The association of persistent pain with out-patient addiction treatment outcomes and service utilization. Addiction. 2008;103(12):1996-2005. doi:10.1111/j.1360-0 443.2008.02358.x

6. Strathdee SA, Palepu A, Cornelisse PG, et al. Barriers to use of free antiretroviral therapy in injection drug users. JAMA. 1998;280 (6):547-549. doi:10.1001/jama.280.6.547

7. Von Korff M, Ormel J, Keefe FJ, Dworkin SF. Grading the severity of chronic pain. Pain. 1992;50(2):133-149. doi:10.1016/0304-3959(92) 90154-4
8. Wang L, Panagiotoglou D, Min JE, et al. Inability to access health and social services associated with mental health among people who inject drugs in a Canadian setting. Drug Alcohol Depend. 2016;168:22-29. doi:10.1016/j.drugalcdep.2016. 08.631

9. Blumenreich PE, Lewis S. Managing the Violent Patient: A Clinician's Guide. New York: Brunner/Mazel; 1993.

10. Wright NM, Dixon CA, Tompkins CN. Managing violence in primary care: an evidence-based approach. Br J Gen Pract. 2003;53 (492):557-562.

11. Schwartz HI, Pinsker H. Mediating retention or release of the potentially dangerous patient. Hosp Community Psychiatry. 1987;38 (1):75-77. doi:10.1176/ps.38.1.75

12. Fitzmaurcie GM, Laird NM, Ware JH. Applied Longitudinal Analysis. New Jersey: John Wiley; 2004.

13. Raudenbush SW, Bryk AS. Hierarchical Linear Models: Applications and Data Analysis Methods. 2nd ed. Thousand Oaks, CA: Sage Publications; 2002.

14. Lang T. Documenting research in scientific articles: guidelines for authors: 3. Reporting multivariate analyses. Chest. 2007;131 (2):628-632. doi:10.1378/chest.06-2088

15. Rosenblum A, Joseph H, Fong C, Kipnis S, Cleland C, Portenoy RK. Prevalence and characteristics of chronic pain among chemically dependent patients in methadone maintenance and residential treatment facilities. JAMA. 2003;289(18):2370-2378. doi:10.1001/ jama.289.18.2370

16. Jamison RN, Kauffman J, Katz NP. Characteristics of methadone maintenance patients with chronic pain. $J$ Pain Symptom Manage. 2000;19(1):53-62. doi:10.1016/S0885-3924(99)00 $144-X$

17. Trafton JA, Oliva EM, Horst DA, Minkel JD, Humphreys K. Treatment needs associated with pain in substance use disorder patients: implications for concurrent treatment. Drug Alcohol Depend. 2004;73(1):23-31. doi:10.1016/j.drugalcdep.2003.08.007

18. Passik SD, Hays L, Eisner N, Kirsh KL. Psychiatric and pain characteristics of prescription drug abusers entering drug rehabilitation. J Pain Palliat Care Pharmacother. 2006;20(2):5-13. doi:10.1080/ J354v20n02_03

19. Worley MJ, Heinzerling KG, Shoptaw S, Ling W. Volatility and change in chronic pain severity predict outcomes of treatment for prescription opioid addiction. Addiction. 2017;112(7):1202-1209. doi:10.1111/add.13782

20. McPherson C, Collins E, Boyne H, Kirkaldy E, Waseem R. Selfreported chronic pain as a predictor of relapse post residential addiction treatment: a 6-month follow-up pilot study. Int J Ment Health Addict. 2017;15(5):1069-1079. doi:10.1007/s11469-0179751-1

21. Larson MJ, Paasche-Orlow M, Cheng DM, Lloyd-Travaglini C, Saitz R, Samet JH. Persistent pain is associated with substance use after detoxification: a prospective cohort analysis. Addiction. 2007;102(5):752-760. doi:10.1111/j.1360-0443.2007.01759.x

22. Savage SR, Kirsh KL, Passik SD. Challenges in using opioids to treat pain in persons with substance use disorders. Addict Sci Clin Pract. 2008;4(2):4-25. doi:10.1151/ascp08424

23. Kapuganti A, Turner T, Thomas CJ. Evaluation of buprenorphine/ naloxone dose and use of sedating supportive medication on treatment outcomes in veterans with opioid use disorder. Ment Health Clin. 2017;7(6):271-275. doi:10.9740/ mhc.2017.11.271

24. Peles E, Schreiber S, Gordon J, Adelson M. Significantly higher methadone dose for methadone maintenance treatment (MMT) patients with chronic pain. Pain. 2005;113(3):340-346. doi:10.1016/ j.pain.2004.11.011

25. Voon P, Hayashi K, Milloy MJ, et al. Pain among high-risk patients on methadone maintenance treatment. J Pain. 2015;16(9):887-894. doi:10.1016/j.jpain.2015.06.003 
26. Voon P, Greer AM, Amlani A, Newman C, Burmeister C, Buxton JA Pain as a risk factor for substance use: a qualitative study of people who use drugs in British Columbia, Canada. Harm Reduct J. 2018;15(1):35. doi:10.1186/s12954-018-0241-y

27. Levesque JF, Harris MF, Russell G. Patient-centred access to health care: conceptualising access at the interface of health systems and populations. Int J Equity Health. 2013;12:18. doi:10.1186/1475-9276$12-18$
28. Broderick JE, Schwartz JE, Vikingstad G, Pribbernow M, Grossman S, Stone AA. The accuracy of pain and fatigue items across different reporting periods. Pain. 2008;139(1):146-157. doi:10.1016/j.pain.2008.03.024

\section{Publish your work in this journal}

The Journal of Pain Research is an international, peer reviewed, open access, online journal that welcomes laboratory and clinical findings in the fields of pain research and the prevention and management of pain. Original research, reviews, symposium reports, hypothesis formation and commentaries are all considered for publication. The manuscript

Submit your manuscript here: https://www.dovepress.com/journal-of-pain-research-journa management system is completely online and includes a very quick and fair peer-review system, which is all easy to use. Visit http:// www.dovepress.com/testimonials.php to read real quotes from published authors. 